\title{
Cross-cultural translation, adaptation and validation of a Japanese version of the Functional Index for Hand Osteoarthritis (J-FIHOA)
}

\section{Yasunobu Nakagawa ( $\nabla$ nyasu756@hotmail.com )}

Nagoya University Graduate School of Medicine https://orcid.org/0000-0002-2628-1336

\section{Shigeru Kurimoto}

Nagoya University Graduate School of Medicine

\section{Emmanuel Maheu}

Department of Rheumatology, AP-HP, Hospital Saint-Antoine

\section{Yuichiro Matsui}

Department of Orthopaedic Surgery, Hokkaido University Graduate School of Medicine

\section{Yuri Kanno}

Yotsuya Medical Cube Hand Surgery and Microsurgery Center

\section{Kunitaka Menuki}

Department of Orthopaedic Surgery, School of Medicine, University of Occupational and Environmental Health

\section{Masanori Hayashi}

Department of Orthopaedic Surgery, Shinshu University School of Medicine

\section{Tetsuya Nemoto}

Department of Orthopaedic Surgery, Showa University School of Medicine

\section{Takanobu Nishizuka}

Nagoya Hand Surgery Center, Chunichi Hospital

\section{Masahiro Tatebe}

Department of Hand Surgery, Nagoya University Graduate School of Medicine

\section{Michiro Yamamoto}

Department of Hand Surgery, Nagoya University Graduate School of Medicine

Katsuyuki Iwatsuki

Department of Hand Surgery, Nagoya University Graduate School of Medicine

\section{Renée Liliane Dreiser}

Department of Rheumatology, AP-HP, Hospital Bichât

\section{Hitoshi Hirata}

Department of Hand Surgery, Nagoya University Graduate School of Medicine 
Keywords: Hand Osteoarthritis, Translation, FIHOA, Japan, Evaluation study, Patient Reported Outcome Measure

Posted Date: January 15th, 2020

DOI: https://doi.org/10.21203/rs.2.20873/v1

License: (c) (1) This work is licensed under a Creative Commons Attribution 4.0 International License. Read Full License

Version of Record: A version of this preprint was published at BMC Musculoskeletal Disorders on March 16th, 2020. See the published version at https://doi.org/10.1186/s12891-020-03193-6. 


\section{Abstract}

Background: Hand osteoarthritis (OA) has a wide spectrum of clinical presentations and physical function is one of the core domains where patients suffer. The Functional Index for Hand Osteoarthritis (FIHOA) is a leading assessment tool for hand dysfunction. Our objective was to make a Japanese version of FIHOA (J-FIHOA) and validate it among Japanese hand OA patients.

Methods: Forward and backward translation processes were completed to create a culturally adapted JFIHOA. A prospective, observational multicenter study was undertaken for the validation process. Seventeen collaborating hospitals recruited Japanese hand OA patients who met the American College of Rheumatology criteria. A medical record review and responses to the following patient-rated questionnaires were collected: J-FIHOA, Hand20, Health Assessment Questionnaire (HAQ), numerical rating scale for pain (NRS pain) and Short Form 36 Health Survey (SF-36). We explored the structure of JFIHOA using factor analysis. Cronbach's alpha coefficients and item-total correlations were calculated. Correlations between J-FIHOA and other questionnaires were evaluated for construct validity. Participants in clinically stable conditions repeated J-FIHOA at a one- to two-week interval to assess test-retest reliability. To evaluate responsiveness, symptomatic patients who started new pharmacological treatments had a 1-month follow-up visit and completed the questionnaires twice. Effect size (ES) and standardized response mean (SRM) were calculated with pre- and post-treatment data sets. We assessed responsiveness, comparing ES and SRM of J-FIHOA with other questionnaires (construct approach).

Results: A total of 210 patients participated. J-FIHOA had unidimensional structure. Cronbach's alphas (0.914 among females and 0.929 among males) and item-total correlations (range, 0.508 to 0.881 ) revealed high internal consistency. Hand20, which measures upper extremity disability, was strongly correlated with J-FIHOA ( $\mathrm{r}=0.82)$ while the mental and role-social component of SF-36 showed no correlations ( $r=-0.24$ and -0.26 , respectively). Intraclass correlation coefficient for test-retest reliability was 0.83 and satisfactory. J-FIHOA showed the highest ES and SRM (-0.68 and -0.62 , respectively) among all questionnaires, except for NRS pain.

Conclusions: Our results showed J-FIHOA had good measurement properties to assess physical dysfunction in Japanese patients both for ambulatory follow-up in clinical practice, and clinical research and therapeutic trials.

\section{Background:}

Hand osteoarthritis (OA) is a highly prevalent and heterogeneous musculoskeletal disorder[1]. Aging increases the risk of its emergence and progression[2]. These issues are especially the case in Japan with its elderly population dramatically growing. While racial differences and gene variants are reported to influence the development of OA, comparatively few studies concern Asian countries[3, 4].

Epidemiologically, the prevalence in Asians such as Japanese and Chinese has been reported to be lower than in white U.S. individuals[5,6]. Yet, a recent study showed a much higher prevalence of radiographic 
hand OA in the Japanese population compared to other cohorts[7-9]. These inconsistencies may be precipitated by heterogeneous presentations and limit the advancement of our understanding of this disease.

Although most Asian studies have focused on radiographic changes of the patients, not all structural damage manifests hand pain or stiffness[10]. A discrepancy exists between the radiographic findings and severity of symptoms[11]. Clinical courses differ in severity, number and distribution of symptomatic joints. There are various hand OA phenotypes that may impact the different outcomes in this disease. Certain patients experience a wide range of deteriorating health conditions, even beyond those with rheumatoid arthritis (RA) $[12,13]$. In addition, some symptoms worsen longitudinally while others improve spontaneously[14]. Physicians and researchers need to distinguish symptomatic from non-symptomatic hand OA patients to both pursue appropriate treatment and engage in future clinical investigations.

Patient reported outcome measures (PROMs) generally play a key role when assessing arthritic conditions such as pain[15], physical dysfunction[16], psychiatric disturbance[17], aesthetic problems[18, $19]$ and poor health-related quality of life[12,13]. While a growing number of instruments are available and cover a wide range of the health sciences, we must use those most appropriate to the clinical setting[20,21]. Since Japan is linguistically not Indo-European and culturally East Asian, we have to consider these differences when introducing new PROMs, many of which were developed in the West[22, 23].

Physical function is one of the core domains in which hand OA patients suffer. The Functional Index for Hand Osteoarthritis (FIHOA) was developed by Dreiser and Maheu in the 1990s and has been a widely adopted PROM in hand OA research[24, 25]. It assesses hand OA-specific disability utilizing a 10 question, 4-point Likert scale (from possible without difficulty to impossible) and has good measurement properties[24-27]. The total score ranges from 0 to 30. A score of at least 5 points has been validated as able to discriminate symptomatic from non-symptomatic hand $O A[24]$. Approximately twenty linguistic versions are available and others are being developed[28-31]. The original English version and relevant references are freely accessible at FIHOA.net. The FIHOA is recommended as an assessment tool for physical dysfunction by the Osteoarthritis Research Society International (OARSI) and European Society on Clinical and Economic Aspects of Osteoporosis, Osteoarthritis and Musculoskeletal Diseases (ESCEO) $[32,33]$. Introducing the FIHOA to Japan will not only stimulate Japanese research but also add to the cumulative knowledge of hand $O A$.

The objective of this study was to create a Japanese version of the FIHOA (J-FIHOA) and to validate it among hand OA patients in Japan where few reliable, validated PROMs for hand OA are currently available.

\section{Methods:}

The original FIHOA assesses hand OA-related functional disability, consisting of 10 questions. We translated and cross-culturally adapted the FIHOA following established guidelines[22, 23]. Subsequently, 
a prospective observational multicenter study was undertaken for the validation process. We evaluated the measurement properties of the J-FIHOA among Japanese hand OA patients within the classical test theory framework, referring to the consensus-based standards for the selection of health measurement instruments (COSMIN) risk of bias checklist to scrutinize the methodological quality of our study[34].

\section{Translation and cultural adaptation}

An expert committee was convened. It was comprised of two language professionals (A.I. and D.S.), three health professionals (Y.N., S.K. and H.H.), and an American native English-speaking research assistant (J.C.). Forward translation was performed independently by one professional translator who had no prior knowledge of the study and the two language professionals serving on the expert committee. All forward translators were native Japanese speakers and fluent in English. Results were assessed and synthesized into a preliminary version of the J-FIHOA. It was translated back into English independently by two English native professional translators, one with a medical background and one without. Both of them were blinded to the study aims and FIHOA concepts throughout the process. Additional forward and backward translations were undertaken to resolve specific points such as linguistic problems. We consulted with the FIHOA developers (E.M. and R.L.D.) about certain discrepancies and issues of interpretation. The translated FIHOA was pretested on 10 Japanese hand OA patients to identify potentially difficult words or phrases. We added kana script above difficult Chinese characters to facilitate comprehension, as is common in written Japanese. We also inserted a question and answer example illustrating how to mark responses. The committee submitted written reports to the developers that documented all processes and how we reached consensus. After their approval, the translation and cultural adaptation process was completed (Table 1).

\section{Validation}

\section{Participant recruitment}

Our university hospital and 16 other hospitals recruited hand OA patients at the outpatient departments from September 2017 to December 2018. New or already followed hand OA patients who were Japanese natives and over 20 years old were eligible. American College of Rheumatology (ACR) classification criteria for hand OA was used for the diagnosis[35]. Patients with other rheumatic diseases or posttraumatic OA were excluded. Participants had conventional therapies for hand OA. Written consent was obtained from each participant. The study protocol was approved by the review board of each participating hospital.

\section{Data collection}

At the enrollment visit a medical history (including duration of hand pain/stiffness and previous treatment for hand $O A$ ), postero-anterior radiographs of both hands and the following patient reported questionnaires were collected: J-FIHOA, Hand20, Japanese version of the Stanford Health Assessment 
Questionnaire (HAQ), numerical rating scale for pain (NRS pain), and Japanese version of the Short Form 36 Health Survey (SF-36).

Participants were followed up to one year to collect longitudinal data sets. For the test-retest reliability, we obtained J-FIHOA data from those whose symptoms and treatment were unchanged over at least 3 months to avoid disease flares or therapeutic modifications. The test-retest interval was one to two weeks. Examinees were allowed to answer the retests either at a face-to-face visit or, for their convenience, via postal mail. To assess the responsiveness, we selected symptomatic hand OA participants who started or changed to certain new systematic pharmacological treatments limited to oral acetaminophen, NSAIDs and/or tramadol. We checked J-FIHOA and other questionnaire scores immediately before the treatment and at a 4-week follow-up visit (+/- 2 weeks). At the follow-up visit, these participants also evaluated the change in the clinical state of their hands using a 7-point Likert scale (global rating of change [GRC]).

\title{
Questionnaires
}

\author{
v J-FIHOA
}

The FIHOA consists of 10 questions, one of which requests a separate response from females, Are you able to sew? and males, Are you able to use a screwdriver? (Question 7). In this study, we removed "for women" and "for men" from Question 7 to obtain all 11 responses regardless of gender. Participants answered all 11 items, from 0 (possible without difficulty) to 3 (impossible), and we calculated the JFIHOA scores in two different ways. One was to sum the 10 items as the original FIHOA does, called the "total score" (range, 0 to 30). Participants with total scores of 5 or more were defined as having symptomatic hand OA. The other was to sum all 11 items, called the "11-item model." We used the 11item model only when performing exploratory factor analysis and pursuing internal consistency on Question 7.

Hand20

The Hand20 is composed of 20 illustrated questions for disorders that assess the upper limb including hands. Scoring for each item ranges from 0 to 10 , higher numbers indicating greater disability. The total score is obtained by dividing the sum of all questions in half (range, 0 to 100). Explanatory illustrations and short, easy-to-understand questions facilitate good response rates especially among elderly people[36, 37].

Japanese version of the Stanford Health Assessment Questionnaire (HAQ)

The HAQ is a widely used instrument to assess functional disability especially in RA[38]. Regarding cultural differences, 3 questions on the Japanese version of HAQ have been modified: get in and out of bed to get up and down from futon, cut your meat to use chopsticks for meal, and a 5 pound object to a 2 liter plastic bottle[39]. Scores are increased to 2 , if they were lower, in any categories in which the patient used a device or relied on help from another person. 
Numerical rating scale for pain (NRS pain)

Global pain of the affected hand(s) was assessed using a numerical rating scale. Participants were asked to mark the level of their pain on a horizontal scale from " 0 = no pain" to "10 = worst pain imaginable."[40]

Japanese version of the Short Form 36 Health Survey (SF-36)

The SF-36 is a questionnaire to measure general health status with 36 questions consisting of eight scales that can be summarized into components. It has been validated in Japanese[41]. A threecomponent model is used when analyzing results of the Japanese version of SF-36 scores: physical component summary (PCS), mental component summary (MCS) and role-social component summary (RCS)[42].

Global rating of change (GRC) scale in clinical state of the hands

Global rating of change (GRC) scales are commonly used to evaluate the responsiveness and to calculate the minimal clinically important change of the scale[43]. We used a GRC scale for symptomatic hand OA patients who started or changed to the new pharmacological treatments. At the 4-week follow-up visit, patients were asked "How would you describe your hand condition compared to before you took the new drug?" and scored their change using a 7-point scale: very much improved, much improved, a little improved, no change, a little deteriorated, much deteriorated, or very much deteriorated. We categorized patients based on the GRC scale and performed subgroup analyses.

\section{Data analysis}

Descriptive analyses were performed to summarize patient characteristics and scores of questionnaires. Incomplete J-FIHOA items were also examined. We compared the characteristics between female and male participants using the Student's t-tests for continuous variables and chi-square tests for categorical variables. By checking the distribution of each questionnaire with the Kolmogorov-Smirnov test, we found that the only measure normally distributed was the SF-36 MCS. We used the Mann-Whitney U test to examine gender difference in each item or total score, and the Spearman's rank correlation coefficients to measure the strength of the associations among items and/or questionnaires. Correlations were categorized as none $(r=0-0.29)$, weak $(r=0.30-0.49)$, moderate $(r=0.50-0.69)$ or strong $(r=0.70-1.00)$. All analyses were carried out using IBM SPSS software, version 24 . The level of significance was set at $p$ values of less than 0.05 .

\section{Validation}

Structural validity

Using the 11-item model, factor analysis was performed with the maximum likelihood method to explore the scale structure of the J-FIHOA. Since the original FIHOA is a hand OA-specific scale, the Japanese 
version was expected to be unidimensional. We determined the number of relevant factors based on eigenvalues larger than one (the Kaiser criterion) and visual inspection of the scree plot[44].

Internal consistency

Internal consistency was evaluated with Cronbach's alphas and correlations between each individual item and the total score of J-FIHOA without it (item-total correlations). To examine gender difference, we compared the score of each item and performed an additional investigation to explore the measurement properties of Questions 7A and 7B, which consist of two gender-role specific items. Cronbach's alphas and item-total correlations were re-calculated in the 11-item model.

Reliability

We selected participants in stable condition, whose symptoms and treatments were unchanged. They were asked to answer the J-FIHOA twice, repeating it after a one- to two-week interval. The intraclass correlation coefficient (ICC) was used to assess test-retest reliability.

Construct validity

To assess construct validity, we performed hypothesis testing by focusing on correlations between the $\mathrm{J}$ FIHOA and the other validated scales. Six hypotheses were established prior to data collection: Hand20 correlation would be the strongest among instruments; HAQ and NRS pain correlations would be moderate; SF-36 PCS correlation would be moderate but MCS and RCS would be weak or none.

\section{Responsiveness}

To analyze responsiveness, we recruited symptomatic participants whose total J-FIHOA scores were 5 or more and who were starting oral analgesic drugs. We used scores immediately before the treatment and at the 4-week follow-up visit (+/- 2 weeks). We compared the scores using the Wilcoxon signed-rank test. The effect size (ES) and standardized response mean (SRM) were also evaluated. ES was obtained by dividing the mean change of scores by the standard deviation (SD) of initial scores. SRM was obtained by dividing the mean change of scores by the SD of that change.

We evaluated responsiveness using two different approaches: comparisons of ES and SRM between the J-FIHOA and other measurements; and subgroup analyses of the J-FIHOA. We expected the J-FIHOA to have the largest ES and SRM among all the PROMs, except for NRS pain. We used the GRC scale for subgroup analysis. At the end of data collection, thirty data sets were available for responsiveness analysis. Almost all patients reported their changes as either a little improved $(n=14)$ or much improved $(n=12)$ and there were no complaints of deterioration. The remaining patients said very much improved $(n=1)$ or no change $(n=3)$. So, we divided the patients into two subgroups based on the GRC scale: major change group (very much improved and much improved, $n=13$ ) and minor change group (a little improved and no change, $n=17$ ). We hypothesized that the major change group would have larger ES and SRM on the J-FIHOA than the minor change group. 


\section{Results:}

\section{Translation}

Most FIHOA items were deemed equivalent in the translation and cultural adaptation process but discrepancies between the original and back translation revealed some issues. On a few major issues, the committee consulted with the FIHOA developers (Supplement A). Considering possible cultural differences, Question 10 Would you accept a handshake without reluctance? was actively debated. The committee decided to translate Question 10 close to the original and explore its rationale in the following validation process.

Descriptive results at enrollment visit

A total of 178 female and 32 male hand OA patients participated (Table 2). Mean age at the enrollment visit was 64.6 years. Approximately $90 \%$ of participants had pain and more than $60 \%$ experienced stiffness. Mean duration of pain and stiffness were both 5.2 years. Female patients had a lower body mass index, lower SF-36 PCS and higher HAQ. Mean total score of J-FIHOA was 5.5 (SD 5.8) and almost half of participants met the definition of symptomatic hand OA. Only two of the 210 participants (1.0\%) failed to answer one or two J-FIHOA items. Questions 4, 7A, and 10 each had one missing response.

Unidimensionality

The eigenvalue of the first factor was 6.59 , which explained the $59.9 \%$ of the total variance in the $\mathrm{J}$ FIHOA. Comparatively, the second factor was 0.86 accounting for only $7.8 \%$ of the variance. The scree plot had a single elbow curve (Fig. 1). These findings confirmed that the J-FIHOA had a unidimensional structure. We also examined the factor loadings, representing associations between each item and the factor, which ranged from 0.61 to 0.85 .

Internal consistency

Cronbach's alpha among females was 0.914 with a $95 \%$ confidence interval (Cl) from 0.893 to 0.931 . Among males it was $0.929(95 \% \mathrm{Cl}, 0.885$ to 960$)$. Item-total correlations were also obtained independently and the values ranged from 0.580 to 0.779 among females and from 0.508 to 0.881 among males (Table 3). No item scores had gender differences, except for Question 4. The item-total correlations of the 11-item model revealed that Question 7B Are you able to use a screwdriver?, originally for men, had the strongest correlation in female participants and vice versa $(0.809$ and 0.885 , respectively).

\section{Test-retest reliability}

One hundred thirty-five data sets were used to evaluate test-retest reliability. Mean J-FIHOA scores were 4.8 (SD 5.7) at the test and 5.5 (SD 5.9) at the retest. The ICC was 0.83 ( $95 \% \mathrm{Cl}, 0.77$ to 0.88 ). 
All six hypotheses were assessed and four correlations fulfilled our expectation: Hand20 (the strongest), NRS pain (moderate), SF-36 MCS (none), and SF-36 RCS (none) (Table 4).

\section{Responsiveness}

Thirty longitudinal data sets were pooled. Before treatment, the mean J-FIHOA score was 11.6 (SD 4.7) and NRS pain was 6.8 (SD 1.8). After oral pharmacological treatments, J-FIHOA and NRS pain decreased by 3.2 and 2.0, respectively. ES and SRM of the J-FIHOA were -0.68 and -0.62 . NRS pain showed a larger ES and SRM than the J-FIHOA did, while smaller ES and SRM were observed on the Hand20 and HAQ (Table 5). We performed subgroup analysis based on the GRC scale. J-FIHOA scores decreased by 4.5 in the major change group and 2.2 in the minor change group. The major change group demonstrated a larger ES and SRM.

\section{Discussion:}

We created the J-FIHOA through a process of forward and backward translations to address the linguistic and cultural differences. We then performed a prospective multicenter study to validate its measurement properties with 210 Japanese hand OA patients with widely ranging conditions from well-controlled to severely symptomatic.

The translation process was performed following guidelines and publications distilled from comparable initiatives[22, 23]. Justifications for cultural adaptation depend on concepts of the instrument and populations concerned. Although the FIHOA consists of simple questions focusing on daily activities, several points needed to be scrutinized, especially the experimental equivalence of Question 10 Would you accept a handshake without reluctance?. As a cultural convention, Japanese, the elderly in particular, shake hands infrequently. Yet, the committee and developers agreed that Question 10 was unique and irreplaceable because it assessed aspects of aesthetics and communication.

Exploratory factor analysis revealed that the J-FIHOA was a unidimensional scale and all loadings satisfied the minimum requirement, usually set at 0.5[44]. All questions contributed to a single factor, which we considered representative of "physical dysfunction." Cronbach's alphas were above 0.9 and each item-total correlation exceeded 0.5. These findings indicated that the J-FIHOA was a unidimensional scale with good internal consistency[45]. Question 10 also showed feasibility and consistency with only one missing response.

We also examined another concern regarding gender differences using the 11-item model[32]. Females had a statistically higher score (i.e. greater disability) in Question 4 Are you able to lift a full bottle with the hand?. We assumed it might be due to inherent differences, such as in muscle strength[46]. Both Questions 7A and 7B had the strongest item-total correlations (i.e. 7A for women, 0.885 among males and $7 \mathrm{~B}$ for men, 0.809 among females), suggesting that the gender specific parts were irrelevant and could be removed. This gave rise to another concern regarding how to treat Question 7-measure both items, delete either, or combine. We were indecisive but disinclined to deviate from the original and kept 
"for women" and "for men" in the J-FIHOA. Additional investigation might be necessary such as differential item functioning (DIF) analysis, which assesses different probabilities of responding to certain items among different groups (ideally with more than 100 patients per group)[34].

Several validated PROMs were used to examine associations with the J-FIHOA. In addition to the FIHOA, the Australian/Canadian Hand OA Index (AUSCAN) is the other hand OA-specific assessment tool[47]. AUSCAN has excellent measurement properties and has been frequently applied to hand OA clinical trials[26, 27, 32]. Although a number of linguistic versions are available, AUSCAN has not been translated and validated into Japanese. In addition, the tool is not freely available. Therefore, we did not include AUSCAN in this study.

Construct validity was assessed by testing hypotheses based on the fundamental assumptions that the FIHOA scores reflected the severity of physical dysfunction of the hands and that the Japanese version was equivalent to validated versions of the FIHOA. Hand20 assesses upper limb dysfunction and has some similar items to the J-FIHOA such as "Do up shirt buttons with both hands" (cf. Question 8 Are you able to fasten buttons?). It showed the strongest correlations $(r=0.82)$, as expected. Although the FIHOA has no pain-related items, pain has been reported to be moderately correlated on the FIHOA and our data were consistent $(r=0.58)[25,24,26,28-31]$. Since mental and social status are dimensions distinct from physical condition, no correlations were observed in the SF-36 MCS and RCS $(r=-0.22$ and -0.23 , respectively). Our results showed that the HAQ had a stronger correlation $(r=0.73)$ and SF-36 PCS had a weaker correlation $(r=-0.36)$ than previous reports, where both HAQ and SF-36 PCS were moderately correlated with the FIHOA ( $r=0.57$ to 0.73 and $r=-0.57$ to -0.67 , respectively) $[26,30,29,31]$. We concluded that our results were not inconsistent with the construct of the J-FIHOA because these correlations largely depended on patient characteristics or conditions.

Longitudinal data enabled an evaluation of the test-retest reliability (135 participants) and responsiveness (30 participants). In the test-retest analysis, we allowed the examinees to answer the retests either at a face-to-face visit or via postal mail. Most chose the latter. Although the different forms of administration might have affected the reliability, the ICC was 0.83 . Even the lower bound of the $95 \% \mathrm{Cl}$ was greater than 0.70 , the minimal requirement of reliability, indicating the J-FIHOA had good test-retest reliability.[44]

ES and SRM are widely used to evaluate responsiveness. However, the COSMIN suggests that both are inappropriate in some situations. One reason is that ES and SRM are highly dependent on the SD of initial scores and change scores, respectively. If the target population is homogeneous or the variation in treatment effect is small, these values can be large. So, we assessed responsiveness of the J-FIHOA by two construct approaches, comparing with other PROMs and dividing the patients into two subgroups[34]. The J-FIHOA showed the highest ES and SRM among all PROMs, except for NRS pain. We assumed that it was because J-FIHOA was a hand OA-specific scale and could detect subtle differences in physical dysfunction. It was unsurprising that NRS pain showed the best responsiveness among the questionnaires. We used oral analgesic drugs with a short duration for assessing responsiveness 
because no disease-modifying drugs are available and evidence does not support surgical intervention, especially for osteoarthritis of interphalangeal joints[48]. Previous clinical trials also revealed that pain scoring is the most sensitive tool in hand OA symptom assessment[49]. Subgroup analysis was performed by clustering the patients into two groups based on the GRC scale. As expected, the patients who reported greater improvements obtained a larger ES and SRM. Although the results indicated that the J-FIHOA had good responsiveness, the number of longitudinal data sets were relatively small $(n=30)$ and inadequate for further analyses such as of minimal clinically important difference[50].

The strengths of this study were the number and diversity of our hand OA patient panels, from wellcontrolled to awaiting scheduled surgery with severe symptoms. They enabled us to estimate precise internal consistency and easily generalize our results to a wide range of clinical and research settings. To our knowledge, with its 17 participating university and community hospitals, this is the first multicenter study conducted for hand OA research in Japan. We are confident that this framework will function effectively in future clinical investigations.

This study has several limitations. We did not evaluate measurement invariance by comparing Japanese and Western patients directly. Some cultural inequalities, such as in daily activity, personality trait and perception of physical dysfunction, may variously have influenced responses to each question. Data from two populations and DIF analysis would reveal differences at item levels. Another limitation is that we have not verified the diagnostic cut-off value of the J-FIHOA for defining symptomatic hand OA among Japanese patients. We neither recruited healthy individuals nor clearly defined non-symptomatic hand OA based on criteria other than the J-FIHOA scores. We anticipate future international trials or Japanese cohort studies will accumulate more evidence regarding the measurement properties of the J-FIHOA.

\section{Conclusions:}

We created the cross-culturally adapted J-FIHOA and validated it among Japanese hand OA patients. Our results show the equivalence with the original version and its advantageous measurement properties for assessing physical dysfunction of the hands. The J-FIHOA has the potential both to benefit clinical practice and to accelerate clinical research and therapeutic trials, by promoting comparisons and syntheses of studies within and outside of Japan.

\section{Abbreviations}

ACR:American College of Rheumatology; AUSCAN:Australian/Canadian Hand OA Index; BMI:Body mass index; Cl:Confidence interval; COSMIN:Consensus-based standards for the selection of health measurement instruments; DIF:Differential item functioning; ES:Effect size; ESCEO:European Society on Clinical and Economic Aspects of Osteoporosis, Osteoarthritis and Musculoskeletal Diseases; FIHOA; Functional Index for Hand Osteoarthritis; GRC:Global rating of change; HAQ:Health Assessment Questionnaire; ICC:Intraclass correlation coefficient; J-FIHOA:Japanese version of the Functional Index for Hand Osteoarthritis; MCS:Mental component summary; NRS:Numerical rating scale; OA:Osteoarthritis; 
OARSI:Osteoarthritis Research Society International; PCS:Physical component summary; PROM:Patient reported outcome measure; RA:Rheumatoid arthritis; RCS:Role-social component summary; SD:Standard deviation; SF-36:Short Form 36 Health Survey; SRM:Standardized response mean

\section{Declarations}

\section{Ethics approval and consent to participate:}

This study was approved by the Institutional Review Board of Nagoya University Hospital (2016-0475-2) and those of participating sites. Written consent was obtained from each participant.

\section{Consent for publication:}

Not applicable

\section{Availability of data and materials:}

The datasets used during the current study are available from the corresponding author on reasonable request.

\section{Conflict of interest:}

All authors declare that they have no conflict of interest.

\section{Funding:}

This work was supported by JSPS KAKENHI Grant Number JP18K16620, an Academic Research Project Grant from the Japanese Society for Surgery of the Hand, and Hand Frontier, a non-profit organization dedicated to researching diseases of the upper extremity (www.handfrontier.org). None were involved in study design, data analysis or interpreting the results.

\section{Author contributions:}

YN developed the study framework and was responsible for data analysis, data interpretation, and drafting the manuscript. $\mathrm{HH}$ and SK participated in designing the study and the translation and cultural adaptation process as expert committee members. EM and RLD, the developers of the FIHOA, contributed to creating and revising the J-FIHOA. YM, YK, KM, MH, T Nemoto, T Nishizuka, MT, MY, and KI contributed to data collection and interpretation. All authors critically reviewed the manuscript and approved the final version.

\section{Acknowledgements:}


I thank Tetsuro Onishi, Toshikazu Kurahashi, Shuichi Kato, Mao Akane, Hiroki Shinkai, Hiroya Kawamoto, Kimitoshi Noto, Kenichi Asano, Hisao Ishii, Hidemasa Yoneda for contribution to data collection. I also thank all of my colleagues of the Department of Hand Surgery at Nagoya University, especially Yoshiko Tomoyama for her dedicated support. I appreciate Akiko Ito, from the Institute of International Education and Exchange, Nagoya University, and Daisuke Suematsu, from the Graduate School of Languages and Literatures, Nagoya University, for taking part in the translation and cultural adaptation process as expert committee members and providing valuable insights. I am deeply grateful to James Curley for his assistance in writing the manuscript and encouraging comments. He also played a key role as an expert committee member.

\section{References}

1. Kloppenburg M, Kwok WY (2012) Hand osteoarthritis-a heterogeneous disorder. Nat Rev Rheumatol 8 (1):22-31. doi:10.1038/nrrheum.2011.170

2. Loeser RF (2013) Aging processes and the development of osteoarthritis. Curr Opin Rheumatol 25 (1):108-113. doi:10.1097/BOR.0b013e32835a9428

3. Zhai G, Van Meurs JB, Livshits G, Meulenbelt I, Valdes AM, Soranzo N, Hart D, Zhang F, Kato BS, Richards JB (2009) A genome-wide association study suggests that a locus within the ataxin 2 binding protein 1 gene is associated with hand osteoarthritis: the Treat-OA consortium. J Med Genet 46 (9):614-616. doi:10.1136/jmg.2009.067314

4. Hamalainen S, Solovieva S, Vehmas T, Luoma K, Leino-Arjas P, Hirvonen A (2014) Genetic Influences on Hand Osteoarthritis in Finnish Women - A Replication Study of Candidate Genes. Plos One 9 (5):11. doi:10.1371/journal.pone.0097417

5. Yoshida S, Aoyagi K, Felson DT, Aliabadi P, Shindo H, Takemoto T (2002) Comparison of the prevalence of radiographic osteoarthritis of the knee and hand between Japan and the United States. J Rheumatol 29 (7):1454-1458

6. Zhang Y, Xu L, Nevitt MC, Niu J, Goggins JP, Aliabadi P, Yu W, Lui LY, Felson DT (2003) Lower prevalence of hand osteoarthritis among Chinese subjects in Beijing compared with white subjects in the United States: the Beijing Osteoarthritis Study. Arthritis Rheum 48 (4):1034-1040. doi:10.1002/art.10928

7. van Saase JL, van Romunde LK, Cats A, Vandenbroucke JP, Valkenburg HA (1989) Epidemiology of osteoarthritis: Zoetermeer survey. Comparison of radiological osteoarthritis in a Dutch population with that in 10 other populations. Ann Rheum Dis 48 (4):271-280. doi:10.1136/ard.48.4.271

8. Dahaghin S, Bierma-Zeinstra SMA, Ginai AZ, Pols HAP, Hazes JMW, Koes BW (2005) Prevalence and pattern of radiographic hand osteoarthritis and association with pain and disability (the Rotterdam study). Ann Rheum Dis 64 (5):682-687. doi:10.1136/ard.2004.023564

9. Kodama R, Muraki S, Oka H, lidaka T, Teraguchi M, Kagotani R, Asai Y, Yoshida M, Morizaki Y, Tanaka S, Kawaguchi H, Nakamura K, Akune T, Yoshimura N (2016) Prevalence of hand osteoarthritis and its 
relationship to hand pain and grip strength in Japan: The third survey of the ROAD study. Mod Rheumatol 26 (5):767-773. doi:10.3109/14397595.2015.1130673

10. Fransen M, Bridgett L, March L, Hoy D, Penserga E, Brooks P (2011) The epidemiology of osteoarthritis in Asia. Int J Rheum Dis 14 (2):113-121. doi:10.1111/j.1756-185X.2011.01608.x

11. Bijsterbosch J, Watt I, Meulenbelt I, Rosendaal FR, Huizinga TWJ, Kloppenburg M (2011) Clinical and radiographic disease course of hand osteoarthritis and determinants of outcome after 6 years. Ann Rheum Dis 70 (1):68-73. doi:10.1136/ard.2010.133017

12. Slatkowsky-Christensen B, Mowinckel P, Loge JH, Kvien TK (2007) Health-related quality of life in women with symptomatic hand osteoarthritis: a comparison with rheumatoid arthritis patients, healthy controls, and normative data. Arthritis Rheum 57 (8):1404-1409. doi:10.1002/art.23079

13. Michon M, Maheu E, Berenbaum F (2011) Assessing health-related quality of life in hand osteoarthritis: a literature review. Ann Rheum Dis 70 (6):921-928. doi:10.1136/ard.2010.131151

14. Aspelund G, Gunnarsdottir S, Jonsson P, Jonsson H (1996) Hand osteoarthritis in the elderly. Application of clinical criteria. Scand J Rheumatol 25 (1):34-36. doi:10.3109/03009749609082665

15. Salaffi F, Stancati A, Silvestri CA, Ciapetti A, Grassi W (2004) Minimal clinically important changes in chronic musculoskeletal pain intensity measured on a numerical rating scale. Eur J Pain 8 (4):283291. doi:10.1016/j.ejpian.2003.09.004

16. McConnell S, Kolopack P, Davis AM (2001) The Western Ontario and McMaster Universities Osteoarthritis Index (WOMAC): a review of its utility and measurement properties. Arthritis Rheum 45 (5):453-461. doi:10.1002/1529-0131(200110)45:5<453::Aid-art365>3.0.Co;2-w

17. Damman W, Liu R, Kaptein AA, Evers AWM, van Middendorp H, Rosendaal FR, Kloppenburg M (2018) Illness perceptions and their association with 2 year functional status and change in patients with hand osteoarthritis. Rheumatology (Oxford) 57 (12):2190-2199. doi:10.1093/rheumatology/key231

18. Hodkinson B, Maheu E, Michon M, Carrat F, Berenbaum F (2012) Assessment and determinants of aesthetic discomfort in hand osteoarthritis. Ann Rheum Dis 71 (1):45-49. doi:10.1136/ard.2011.153965

19. Neuprez A, Bruyere O, Maheu E, Dardenne N, Burlet N, D'Hooghe P, Disteche S, Reginster JY (2015) Aesthetic discomfort in hand osteoarthritis: results from the Llege Hand Osteoarthritis Cohort (LIHOC). Arthritis Res Ther 17:346. doi:10.1186/s13075-015-0807-y

20. Garratt AN, Schmidt L, Mackintosh A, Fitzpatrick R (2002) Quality of life measurement: bibliographic study of patient assessed health outcome measures. BMJ 324 (7351):1417-1419. doi:10.1136/bmj.324.7351.1417

21. Maheu E, Berenbaum F (2009) Time for new outcome measures in hand osteoarthritis? Nat Clin Pract Rheumatol 5 (3):136-138. doi:10.1038/ncprheum1021

22. Guillemin F, Bombardier C, Beaton D (1993) Cross-cultural adaptation of health-related quality of life measures: literature review and proposed guidelines. J Clin Epidemiol 46 (12):1417-1432. doi:10.1016/0895-4356(93)90142-n 
23. Beaton DE, Bombardier C, Guillemin F, Ferraz MB (2000) Guidelines for the process of cross-cultural adaptation of self-report measures. Spine (Phila Pa 1976) 25 (24):3186-3191.

doi:10.1097/00007632-200012150-00014

24. Dreiser RL, Maheu E, Guillou GB, Caspard H, Grouin JM (1995) Validation of an algofunctional index for osteoarthritis of the hand. Rev Rhum Engl Ed 62 (6 Suppl 1):43s-53s

25. Dreiser RL, Maheu E, Guillou GB (2000) Sensitivity to change of the functional index for hand osteoarthritis. Osteoarthritis Cartilage 8:S25-28. doi:10.1053/joca.2000.0332

26. Moe RH, Garratt A, Slatkowsky-Christensen B, Maheu E, Mowinckel P, Kvien TK, Kjeken I, Hagen KB, Uhlig T (2010) Concurrent evaluation of data quality, reliability and validity of the Australian/Canadian Osteoarthritis Hand Index and the Functional Index for Hand Osteoarthritis. Rheumatology (Oxford) 49 (12):2327-2336. doi:10.1093/rheumatology/keq219

27. Visser AW, Boyesen P, Haugen IK, Schoones JW, van der Heijde DM, Rosendaal FR, Kloppenburg M (2015) Instruments Measuring Pain, Physical Function, or Patient's Global Assessment in Hand Osteoarthritis: A Systematic Literature Search. J Rheumatol 42 (11):2118-2134. doi:10.3899/jrheum.141228

28. Wittoek R, Cruyssen BV, Maheu E, Verbruggen G (2009) Cross-cultural adaptation of the Dutch version of the Functional Index for Hand Osteoarthritis (FIHOA) and a study on its construct validity. Osteoarthritis Cartilage 17 (5):607-612. doi:10.1016/j.joca.2008.10.006

29. Gandini F, Giannitti C, Fattore G, Giordano N, Galeazzi M, Fioravanti A (2012) Validation of an Italian version of the functional index for hand osteoarthritis (FIHOA). Mod Rheumatol 22 (5):758-765. doi:10.1007/s10165-011-0579-4

30. Yoosefinejad AK, Motealleh A, Babakhani M (2017) Evaluation of validity and reliability of the Persian version of the functional index of hand osteoarthritis. Rheumatol Int 37 (5):719-725. doi:10.1007/s00296-016-3645-6

31. Ahn GY, Cho SK, Cha SJ, Nam E, Lee JE, Dreiser RL, Maheu E, Sung YK (2018) Cross-cultural adaptation and validation of the Korean Version of the Functional Index for Hand Osteoarthritis (FIHOA). Int J Rheum Dis 21:2095-2103. doi:10.1111/1756-185x.13412

32. Kloppenburg M, Maheu E, Kraus VB, Cicuttini F, Doherty M, Dreiser RL, Henrotin Y, Jiang GL, Mandl L, Martel-Pelletier J, Nelson AE, Neogi T, Pelletier JP, Punzi L, Ramonda R, Simon LS, Wang S, Recommen OHCT (2015) OARSI Clinical Trials Recommendations: Design and conduct of clinical trials for hand osteoarthritis. Osteoarthritis Cartilage 23 (5):772-786. doi:10.1016/j.joca.2015.03.007

33. Reginster JL, Arden NK, Haugen IK, Rannou F, Cavalier E, Bruyere O, Branco J, Chapurlat R, Collaud Basset S, Al-Daghri NM, Dennison EM, Herrero-Beaumont G, Laslop A, Leeb BF, Maggi S, Mkinsi O, Povzun AS, Prieto-Alhambra D, Thomas T, Uebelhart D, Veronese N, Cooper C (2018) Guidelines for the conduct of pharmacological clinical trials in hand osteoarthritis: Consensus of a Working Group of the European Society on Clinical and Economic Aspects of Osteoporosis, Osteoarthritis and Musculoskeletal Diseases (ESCEO). Semin Arthritis Rheum 48 (1):1-8.

doi:10.1016/j.semarthrit.2017.12.003

Page 16/20 
34. Mokkink LB, De Vet HC, Prinsen CA, Patrick DL, Alonso J, Bouter LM, Terwee CB (2018) COSMIN risk of bias checklist for systematic reviews of patient-reported outcome measures. Qual Life Res 27 (5):1171-1179. doi:10.1007/s11136-017-1765-4

35. Altman R, Alarcon G, Appelrouth D, Bloch D, Borenstein D, Brandt K, Brown C, Cooke TD, Daniel W, Gray R, Greenwald R, Hochberg M, Howell D, Ike R, Kapila P, Kaplan D, Koopman W, Longley S, McShane DJ, Medsger T, Michel B, Murphy W, Osial T, Ramseygoldman R, Rothschild B, Stark K, Wolfe $F$ (1990) The American College of Rheumatology criteria for the classification and reporting of osteoarthritis of the hand. Arthritis Rheum 33 (11):1601-1610. doi:10.1002/art.1780331101

36. Suzuki M, Kurimoto S, Shinohara T, Tatebe M, Imaeda T, Hirata H (2010) Development and validation of an illustrated questionnaire to evaluate disabilities of the upper limb. J Bone Joint Surg Br 92B (7):963-969. doi:10.1302/0301-620x.92b7.23410

37. Kurimoto S, Yamamoto M, Shinohara T, Tatebe M, Katsuyuki I, Hirata H (2013) Favorable effects of explanatory illustrations attached to a self-administered questionnaire for upper extremity disorders. Qual Life Res 22 (5):1145-1149. doi:10.1007/s11136-012-0233-4

38. Bruce B, Fries JF (2003) The Stanford Health Assessment Questionnaire: A review of its history, issues, progress, and documentation. J Rheumatol 30 (1):167-178

39. Matsuda Y, Singh G, Yamanaka H, Tanaka E, Urano W, Taniguchi A, Saito T, Hara M, Tomatsu T, Kamatani N (2003) Validation of a Japanese version of the Stanford Health Assessment Questionnaire in 3,763 patients with rheumatoid arthritis. Arthritis Rheum 49 (6):784-788. doi:10.1002/art.11465

40. Farrar JT, Young JP, LaMoreaux L, Werth JL, Poole RM (2001) Clinical importance of changes in chronic pain intensity measured on an 11-point numerical pain rating scale. Pain 94 (2):149-158. doi:10.1016/s0304-3959(01)00349-9

41. Fukuhara S, Bito S, Green J, Hsiao A, Kurokawa K (1998) Translation, adaptation, and validation of the SF-36 Health Survey for use in Japan. J Clin Epidemiol 51 (11):1037-1044. doi:10.1016/S08954356(98)00095-X

42. Suzukamo Y, Fukuhara S, Green J, Kosinski M, Gandek B, Ware JE (2011) Validation testing of a three-component model of Short Form-36 scores. J Clin Epidemiol 64 (3):301-308. doi:10.1016/j.jclinepi.2010.04.017

43. Kamper SJ, Maher CG, Mackay G (2009) Global rating of change scales: a review of strengths and weaknesses and considerations for design. J Man Manip Ther 17 (3):163-170. doi:10.1179/jmt.2009.17.3.163

44. de Vet HC, Terwee CB, Mokkink LB, Knol DL (2011) Measurement in medicine: a practical guide. Cambridge University Press, Cambridge, UK

45. Ponterotto JG, Ruckdeschel DE (2007) An overview of coefficient alpha and a reliability matrix for estimating adequacy of internal consistency coefficients with psychological research measures. Percept Mot Skills 105 (3 Pt 1):997-1014. doi:10.2466/pms.105.3.997-1014 
46. Kennedy D, Stratford PW, Pagura SM, Walsh M, Woodhouse LJ (2002) Comparison of gender and group differences in self-report and physical performance measures in total hip and knee arthroplasty candidates. J Arthroplasty 17 (1):70-77. doi:10.1054/arth.2002.29324

47. Bellamy N, Campbell J, Haraoui B, Buchbinder R, Hobby K, Roth JH, MacDermid JC (2002) Dimensionality and clinical importance of pain and disability in hand osteoarthritis: Development of the Australian/Canadian (AUSCAN) Osteoarthritis Hand Index. Osteoarthritis Cartilage 10 (11):855862. doi:10.1053/joca.2002.0837

48. Kloppenburg M, Kroon FP, Blanco FJ, Doherty M, Dziedzic KS, Greibrokk E, Haugen IK, HerreroBeaumont G, Jonsson H, Kjeken I, Maheu E, Ramonda R, Ritt MJ, Smeets W, Smolen JS, Stamm TA, Szekanecz Z, Wittoek R, Carmona L (2019) 2018 update of the EULAR recommendations for the management of hand osteoarthritis. Ann Rheum Dis 78 (1):16-24. doi:10.1136/annrheumdis-2018213826

49. Kvien TK, Fjeld E, Slatkowsky-Christensen B, Nichols M, Zhang Y, Proven A, Mikkelsen K, Palm O, Borisy AA, Lessem J (2008) Efficacy and safety of a novel synergistic drug candidate, CRx-102, in hand osteoarthritis. Ann Rheum Dis 67 (7):942-948. doi:10.1136/ard.2007.074401

50. Tubach F, Ravaud P, Martin-Mola E, Awada H, Bellamy N, Bombardier C, Felson DT, Hajjaj-Hassouni N, Hochberg M, Logeart I, Matucci-Cerinic M, van de Laar M, van der Heijde D, Dougados M (2012) Minimum clinically important improvement and patient acceptable symptom state in pain and function in rheumatoid arthritis, ankylosing spondylitis, chronic back pain, hand osteoarthritis, and hip and knee osteoarthritis: Results from a prospective multinational study. Arthritis Care Res (Hoboken) 64 (11):1699-1707. doi:10.1002/acr.21747

\section{Tables}

Due to technical limitations, Tables 1-5 are provided in the Supplementary Files section.

\section{Figures}




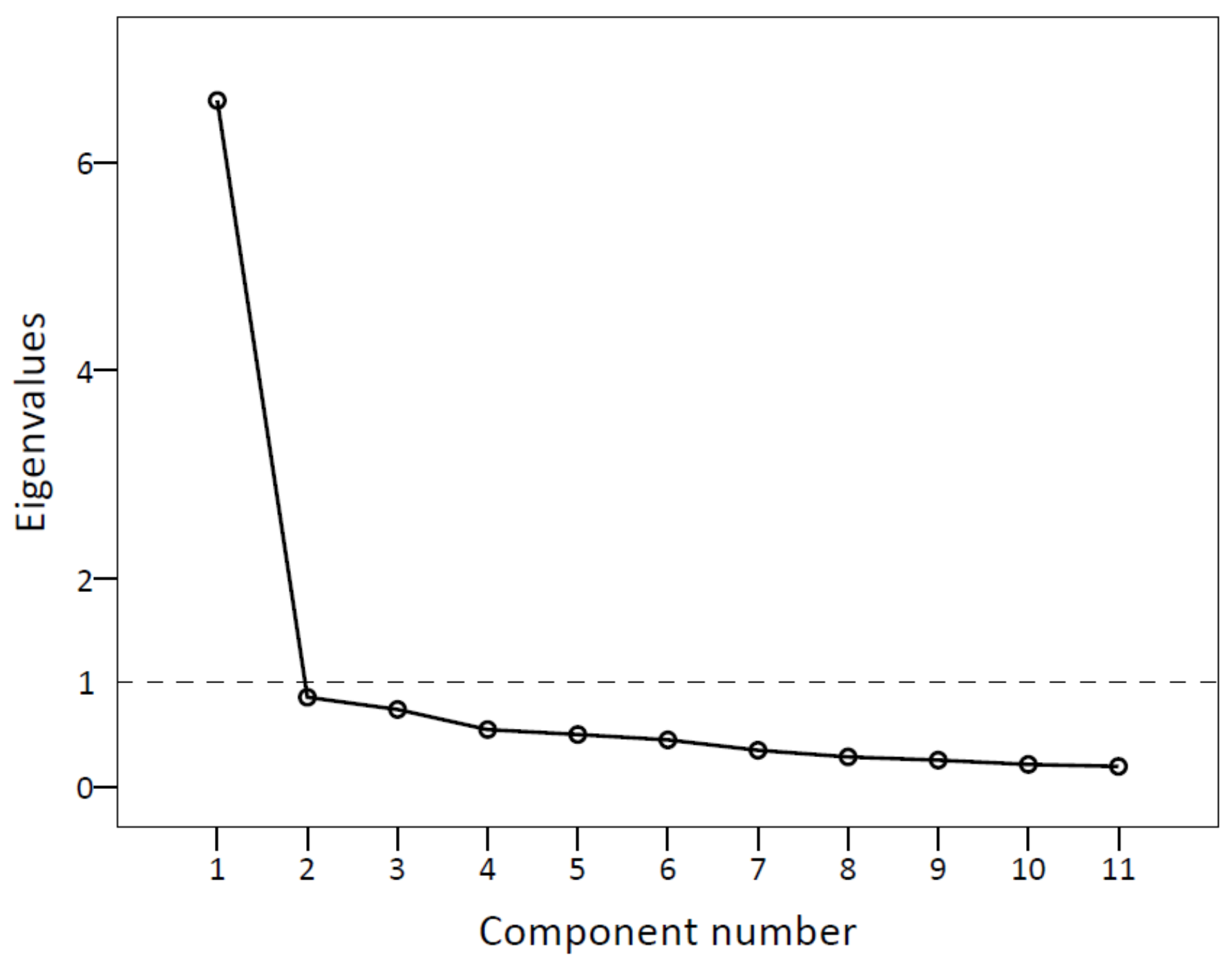

Figure 1

Scree plot of J-FIHOA. The eigenvalue for the first factor was greater than one and accounted for almost $60 \%$ of the total variance. The single elbow in the scree plot also indicated that the J-FIHOA was a unidimensional scale.

\section{Supplementary Files}

This is a list of supplementary files associated with this preprint. Click to download.

- table3.png

- table5.png

- SupplementAJFIHOANakagawa.PDF

- table1.png 
- table2.png

- table4.png 